\title{
Development and characterization of novel expressed sequence tag-derived simple sequence repeat markers in Hevea brasiliensis (rubber tree)
}

\author{
Z.W. An ${ }^{1,2}$, Y.C. Li ${ }^{1}$, Q.L. Zhai ${ }^{1}$, L.L. Xie ${ }^{1}$, Y.H. Zhao ${ }^{3}$ and H.S. Huang ${ }^{2}$ \\ ${ }^{1}$ College of Agriculture, Hainan University, Haikou, Hainan, China \\ ${ }^{2}$ State Centre for Rubber Breeding \& Rubber Research Institute, \\ Chinese Academy of Tropical Agricultural Sciences, Danzhou, Hainan, China \\ ${ }^{3}$ College of Life Sciences, Ludong University, Yantai, Shangdong, China \\ Corresponding author: H.S. Huang \\ E-mail: azwcn@126.com
}

Genet. Mol. Res. 12 (4): 5905-5910 (2013)

Received March 13, 2013

Accepted September 8, 2013

Published November 22, 2013

DOI http://dx.doi.org/10.4238/2013.November.22.18

\begin{abstract}
Cultivated clones of Hevea brasiliensis have a narrow genetic base. In order to broaden the genetic base, it is first necessary to investigate the genetic diversity of wild populations. Expressed sequence tag-simple sequence repeat (EST-SSR) markers were developed to investigate the genetic diversity of Hevea populations. Four hundred and thirty microsatellites were identified and 148 primers were designed to amplify the loci. Twenty-nine primer pairs were synthesized and evaluated for their ability to detect genetic polymorphisms among 40 wild accessions of $H$. brasiliensis. Twenty-one of the 29 loci were polymorphic. The number of alleles per locus in the 40 accessions ranged from 2 to $7 . H_{\mathrm{O}}$ and $H_{\mathrm{E}}$ at each locus ranged from 0.0000 to 0.9000 and from 0.0000 to 0.8704 , respectively. All 21 loci could amplify in $H$. brasiliensis, $H$. pauciflora, $H$. nitida, $H$. spruceana, and $H$. camargoana. The EST-SSR primers developed herein can be used in genetic diversity and structure studies in $H$. brasiliensis.
\end{abstract}

Key words: Hevea brasiliensis; Expressed sequence tags; Microsatellite; Diversity 


\section{INTRODUCTION}

Hevea brasiliensis (rubber tree), the main source of natural rubber, is indigenous to the Amazon basin of South America. Today, the rubber tree is extensively cultivated in Southeast Asia, where it comprises more than $90 \%$ of the world's natural rubber production (Priyadarshan and Gonçalves, 2003). The cultivated clones of Southeast Asia and China were all derived from 22 seedlings surviving from seeds collected by Henry Wickham in 1876, which showed little genetic variation (Tan, 1987).

Expressed sequence tag-derived simple sequence repeat (EST-SSR) markers are powerful tools for many purposes, such as genetic diversity, linkage map, and marker-assisted selection, and can be used across a number of related species. EST-SSRs are developed from ESTs. Because mRNA expressions differ among different tissues or developmental phases, several different EST-SSRs can be identified. Although EST-SSR markers have been developed for rubber tree (Feng et al., 2009; Triwitayakorn et al., 2011; Li et al., 2012), novel SSR markers are still needed for rubber tree research. Rubber trees show little genetic variation (Tan, 1987), and therefore, a lot of SSR markers that are distributed throughout the rubber tree genome are needed to accurately analyze genetic structure, construct a high-density genetic map, or identify linkage markers, which can then facilitate the establishment of rubber tree improvement and conservation programs.

\section{MATERIAL AND METHODS}

Forty wild $H$. brasiliensis accessions (Table 1), originating from the Brazilian States of Acre (AC), Rondônia (RO), and Mato Grosso (MT), and four related species (H. pauciflora, H. nitida, H. spruceana, and H. camargoana) were selected from the State Rubber Tree Germplasm Repository of China. Hevea ESTs were obtained via the ENTREZ search tool of the EST database of the National Center for Biotechnology Information (NCBI; http://www. ncbi.nlm.nih.gov/dbEST/). The EST-trimmer software (http://pgrc.ipk-gatersleben.de/misa/ download/est trimmer.pl) was used to remove poly-A or poly-T stretches of the 5'- or 3'-end until there were no $(\mathrm{T})_{6}$ or $(\mathrm{A})_{6}$ within the range of $50 \mathrm{bp}$. ESTs were clustered and assembled into unigenes, including contigs and singletons, using the CAP3 software (Huang and Madan, 1999). After pre-treatment, the SSRIT program (Temnykh et al., 2001) was used to search for di-, tri-, tetra-, penta-, and hexa-nucleotide motifs in rubber unigenes, with a minimum number of five repeats. Primers were designed to amplify these repeats using the Primer Premier 6.1 program (PREMIER Biosoft International, Palo Alto, CA, USA) if the microsatellite repeat sequences were longer than $20 \mathrm{bp}$.

Genomic DNA was extracted from leaf tissues using the protocol described by An and Huang (2005). Polymerase chain reactions (PCR) were carried out with a Mastercycler thermocycler (Eppendorf AG, Hamburg, Germany) in a 10- $\mu \mathrm{L}$ volume containing $20 \mathrm{ng}$ template DNA, $0.2 \mu \mathrm{M}$ of each primer, and $1 \mathrm{X}$ PCR MasterMix (TianGen Biotech, Beijing, China). PCR conditions were as follows: $94^{\circ} \mathrm{C}$ for 5 min followed by 35 cycles of $94^{\circ} \mathrm{C}$ for $1 \mathrm{~min}, 45$ $\mathrm{s}$ at the annealing temperature of each primer pair (Table 1), and $72^{\circ} \mathrm{C}$ for $1 \mathrm{~min}$, with a final extension for $7 \mathrm{~min}$ at $72^{\circ} \mathrm{C}$. The products were electrophoresed on $6 \%$ sequencing polyacrylamide gels (BioRad Sequi-Gen GT, USA), and bands were visualized by silver nitrate staining (An et al., 2009). 


\begin{tabular}{|c|c|c|c|c|c|c|c|c|c|}
\hline No. & Accession ID & Origin & Longitude (W) & Latitude (S) & No. & Accession ID & Origin & Longitude (W) & Latitude (S) \\
\hline 1 & $\mathrm{AC} / \mathrm{S} / 10$ 37/93 & $\mathrm{AC}$ & $68^{\circ} 39^{\prime}$ & $09^{\circ} 04^{\prime}$ & 21 & RO/C/8 24/10 & RO & $62^{\circ} 51^{\prime}$ & $08^{\circ} 02^{\prime}$ \\
\hline 2 & $\mathrm{AC} / \mathrm{X} / 2164 / 221$ & $\mathrm{AC}$ & $68^{\circ} 29^{\prime}$ & $10^{\circ} 38^{\prime}$ & 22 & $\mathrm{RO} / \mathrm{C} / 8$ 24/293 & RO & $62^{\circ} 51^{\prime}$ & $08^{\circ} 02^{\prime}$ \\
\hline 3 & $\mathrm{AC} / \mathrm{F} / 5$ 21/197 & $\mathrm{AC}$ & $70^{\circ} 21^{\prime}$ & $08^{\circ} 10^{\prime}$ & 23 & $\mathrm{RO} / \mathrm{PB} / 2$ 3/82 & RO & $63^{\circ} 34^{\prime}$ & $11^{\circ} 30^{\prime}$ \\
\hline 4 & $\mathrm{AC} / \mathrm{S} / 12$ 42/494 & $\mathrm{AC}$ & $68^{\circ} 39^{\prime}$ & $09^{\circ} 04^{\prime}$ & 24 & $\mathrm{RO} / \mathrm{JP} / 3$ 22/189 & RO & $61^{\circ} 56^{\prime}$ & $10^{\circ} 53^{\prime}$ \\
\hline 5 & $\mathrm{AC} / \mathrm{X} / 2164 / 177$ & $\mathrm{AC}$ & $68^{\circ} 29^{\prime}$ & $10^{\circ} 38^{\prime}$ & 25 & $\mathrm{RO} / \mathrm{PB} / 2$ 3/267 & RO & $63^{\circ} 34^{\prime}$ & $11^{\circ} 30^{\prime}$ \\
\hline 6 & $\mathrm{AC} / \mathrm{AB} / 15-54 / 418$ & $\mathrm{AC}$ & $69^{\circ} 34^{\prime}$ & $10^{\circ} 56^{\prime}$ & 26 & $\mathrm{RO} / \mathrm{C} / 8$ 24/97 & RO & $62^{\circ} 51^{\prime}$ & $08^{\circ} 02^{\prime}$ \\
\hline 7 & $\mathrm{AC} / \mathrm{F} / 521 / 108$ & $\mathrm{AC}$ & $70^{\circ} 21^{\prime}$ & $08^{\circ} 10^{\prime}$ & 27 & $\mathrm{RO} / \mathrm{PB} / 2$ 3/184 & RO & $63^{\circ} 34^{\prime}$ & $11^{\circ} 30^{\prime}$ \\
\hline 8 & $\mathrm{AC} / \mathrm{F} / 5$ 21/203 & $\mathrm{AC}$ & $70^{\circ} 21^{\prime}$ & $08^{\circ} 10^{\prime}$ & 28 & $\mathrm{MT} / \mathrm{IT} / 1831 / 125$ & MT & $56^{\circ} 55^{\prime}$ & $12^{\circ} 40^{\prime}$ \\
\hline 9 & $\mathrm{AC} / \mathrm{T} / 15 / 8$ & $\mathrm{AC}$ & $70^{\circ} 45^{\prime}$ & $08^{\circ} 08^{\prime}$ & 29 & $\mathrm{MT} / \mathrm{IT} / 16-34 / 5$ & MT & $56^{\circ} 55^{\prime}$ & $12^{\circ} 40^{\prime}$ \\
\hline 10 & $\mathrm{AC} / \mathrm{T} / 24 / 67$ & $\mathrm{AC}$ & $70^{\circ} 45^{\prime}$ & $08^{\circ} 08^{\prime}$ & 30 & $\mathrm{MT} / \mathrm{C} / 2$ 10/54 & MT & $56^{\circ} 55^{\prime}$ & $12^{\circ} 40^{\prime}$ \\
\hline 11 & $\mathrm{AC} / \mathrm{T} / 15 / 130$ & $\mathrm{AC}$ & $70^{\circ} 45^{\prime}$ & $08^{\circ} 08^{\prime}$ & 31 & $\mathrm{MT} / \mathrm{C} / 2$ 10/8 & MT & $56^{\circ} 55^{\prime}$ & $12^{\circ} 40^{\prime}$ \\
\hline 12 & $\mathrm{AC} / \mathrm{F} / 5$ 21/220 & $\mathrm{AC}$ & $70^{\circ} 21^{\prime}$ & $08^{\circ} 10^{\prime}$ & 32 & $\mathrm{MT} / \mathrm{C} / 9$ 15/33 & MT & $56^{\circ} 55^{\prime}$ & $12^{\circ} 40^{\prime}$ \\
\hline 13 & $\mathrm{AC} / \mathrm{F} / 5$ 21/100 & $\mathrm{AC}$ & $70^{\circ} 21^{\prime}$ & $08^{\circ} 10^{\prime}$ & 33 & $\mathrm{MT} / \mathrm{C} / 4$ 7/153 & MT & $56^{\circ} 55^{\prime}$ & $12^{\circ} 40^{\prime}$ \\
\hline 14 & $\mathrm{AC} / \mathrm{T} / 24 / 85$ & $\mathrm{AC}$ & $70^{\circ} 45^{\prime}$ & $08^{\circ} 08^{\prime}$ & 34 & $\mathrm{MT} / \mathrm{C} / 2$ 10/155 & MT & $56^{\circ} 55^{\prime}$ & $12^{\circ} 40^{\prime}$ \\
\hline 15 & $\mathrm{RO} / \mathrm{CM} / 10-44 / 738$ & RO & $64^{\circ} 13^{\prime}$ & $12^{\circ} 24^{\prime}$ & 35 & $\mathrm{MT} / \mathrm{C} / 11$ 9/10 & MT & $56^{\circ} 55^{\prime}$ & $12^{\circ} 40^{\prime}$ \\
\hline 16 & $\mathrm{RO} / \mathrm{CM} / 10 / 44 / 683$ & RO & $64^{\circ} 13^{\prime}$ & $12^{\circ} 24^{\prime}$ & 36 & $\mathrm{MT} / \mathrm{C} / 11$ 9/1 & MT & $56^{\circ} 55^{\prime}$ & $12^{\circ} 40^{\prime}$ \\
\hline 17 & $\mathrm{RO} / \mathrm{J} / 5-33 / 38$ & RO & $62^{\circ} 28^{\prime}$ & $10^{\circ} 25^{\prime}$ & 37 & $\mathrm{MT} / \mathrm{C} / 2$ 10/3 & MT & $56^{\circ} 55^{\prime}$ & $12^{\circ} 40^{\prime}$ \\
\hline 18 & $\mathrm{RO} / \mathrm{OP} / 4$ 20/125 & RO & $62^{\circ} 15^{\prime}$ & $10^{\circ} 42^{\prime}$ & 38 & $\mathrm{MT} / \mathrm{C} / 11$ 9/66 & MT & $56^{\circ} 55^{\prime}$ & $12^{\circ} 40^{\prime}$ \\
\hline 19 & $\mathrm{RO} / \mathrm{C} / 9$ 23/238 & RO & $62^{\circ} 51^{\prime}$ & $08^{\circ} 02^{\prime}$ & 39 & $\mathrm{MT} / \mathrm{C} / 11$ 9/70 & MT & $56^{\circ} 55^{\prime}$ & $12^{\circ} 40^{\prime}$ \\
\hline 20 & $\mathrm{RO} / \mathrm{C} / 824 / 272$ & RO & $62^{\circ} 51^{\prime}$ & $08^{\circ} 02^{\prime}$ & 40 & $\mathrm{MT} / \mathrm{C} / 11$ 9/67 & MT & $56^{\circ} 55^{\prime}$ & $12^{\circ} 40^{\prime}$ \\
\hline
\end{tabular}

$\mathrm{AC}=$ Acre; $\mathrm{RO}=$ Rondônia; $\mathrm{MT}=$ Mato Grosso.

The genetic statistics based on three populations were calculated using POPGENE (Yeh and Boyle, 1997), including the number of alleles, observed $\left(H_{\mathrm{O}}\right)$, and expected $\left(H_{\mathrm{E}}\right)$ heterozygosities.

\section{RESULTS AND DISCUSSION}

In total, 430 SSRs were identified from 3090 unigenes, with 66 unigenes containing more than one SSR and 287 unigenes containing only one SSR. The frequency of EST-SSRs observed in the $H$. brasiliensis transcriptome was $11.42 \%$. A total of 148 primer sets were designed. To evaluate the efficiency of primer design and utilization, 29 of the 148 primers were randomly selected and synthesized from the Sangon Company (Shanghai, China), and the genetic diversity of 40 wild accessions were investigated. Among the 29 primer pairs, 8 failed to amplify, and the remaining 21 yielded polymorphism in 40 accessions (Table 2). $H_{\mathrm{O}}$ and $H_{\mathrm{E}}$ at each locus ranged from 0.0000 to 0.9000 and from 0.0000 to 0.8704 , respectively (Table 3). The 21 EST sequences containing SSR loci were blasted against the GenBank non-redundant database using BLASTx (Altschul et al., 1997). Fifteen of the EST sequences showed significant similarities to known genes (Table 2).

The number of alleles per locus in the 40 wild accessions ranged from 2 to 7 , with an average of 3.43, which exceeded the average of 3.12 alleles reported by Feng et al. (2009), but was below the average of 3.85 alleles reported by Triwitayakorn et al. (2011). To test the crossspecies transferability, 21 primer pairs were used for amplification in five Hevea species: $H$. brasiliensis, $H$. pauciflora, $H$. nitida, $H$. spruceana, and $H$. camargoana. The expected PCR products of 21 primer pairs were obtained from all five species (Figure 1).

The primers developed in this study are powerful and suitable for evaluating the genetic diversity and structure of rubber tree populations. These newly developed EST-SSRs are an additional resource for the genetic characterization of $H$. brasiliensis. 


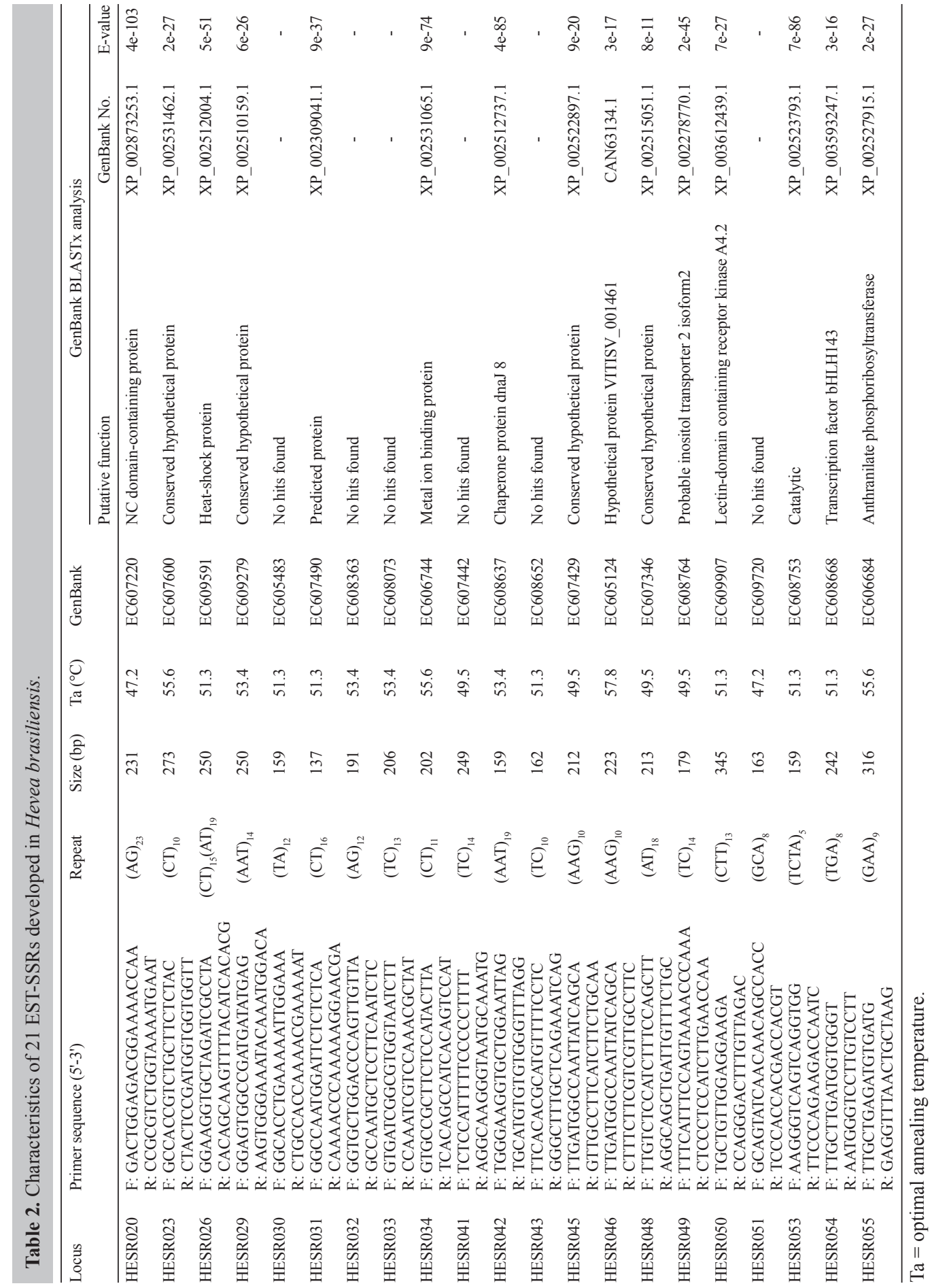


Table 3. Results of initial primer screening in three populations of Hevea brasiliensis.

\begin{tabular}{|c|c|c|c|c|c|c|c|c|c|}
\hline \multirow[t]{2}{*}{ Locus } & \multicolumn{3}{|c|}{$\mathrm{AC}(\mathrm{N}=14)$} & \multicolumn{3}{|c|}{$\mathrm{RO}(\mathrm{N}=13)$} & \multicolumn{3}{|c|}{ MT $(\mathrm{N}=13)$} \\
\hline & $N_{\mathrm{A}}$ & $H_{\mathrm{O}}$ & $H_{\mathrm{E}}$ & $N_{\mathrm{A}}$ & $H_{\mathrm{o}}$ & $H_{\mathrm{E}}$ & $N_{\mathrm{A}}$ & $H_{\mathrm{O}}$ & $H_{\mathrm{E}}$ \\
\hline HESR020 & 2 & 0.2143 & 0.3042 & 2 & 0.4615 & 0.3692 & 2 & 0.5000 & 0.4524 \\
\hline HESR023 & 2 & 0.0000 & 0.2540 & 2 & 0.1538 & 0.5169 & 2 & 0.0000 & 0.2540 \\
\hline HESR026 & 4 & 0.4286 & 0.4683 & 4 & 0.6154 & 0.5169 & 4 & 0.5000 & 0.5476 \\
\hline HESR029 & 7 & 0.6429 & 0.8333 & 6 & 0.6154 & 0.8369 & 7 & 0.7857 & 0.8704 \\
\hline HESR030 & 3 & 0.7857 & 0.6032 & 3 & 0.3846 & 0.5415 & 3 & 0.5714 & 0.5397 \\
\hline HESR031 & 2 & 0.1429 & 0.1376 & 2 & 0.2308 & 0.2123 & 2 & 0.2857 & 0.2540 \\
\hline HESR032 & 6 & 0.7500 & 0.7572 & 6 & 0.9000 & 0.8474 & 4 & 0.9000 & 0.7737 \\
\hline HESR033 & 3 & 0.1429 & 0.3704 & 4 & 0.3846 & 0.5446 & 3 & 0.4286 & 0.5185 \\
\hline HESR034 & 4 & 0.6000 & 0.7842 & 4 & 0.6250 & 0.7917 & 4 & 0.7778 & 0.7647 \\
\hline HESR041 & 3 & 0.5000 & 0.4153 & 1 & 0.0000 & 0.0000 & 2 & 0.4286 & 0.3492 \\
\hline HESR042 & 2 & 0.2857 & 0.2540 & 2 & 0.3077 & 0.2708 & 2 & 0.0714 & 0.0714 \\
\hline HESR043 & 4 & 0.5714 & 0.5476 & 4 & 0.6923 & 0.6277 & 4 & 0.5714 & 0.5899 \\
\hline HESR045 & 2 & 0.6429 & 0.4947 & 2 & 0.6154 & 0.5169 & 2 & 0.4286 & 0.5079 \\
\hline HESR046 & 4 & 0.7143 & 0.7434 & 3 & 0.6923 & 0.5138 & 2 & 0.6429 & 0.4524 \\
\hline HESR048 & 4 & 0.4286 & 0.6667 & 2 & 0.2308 & 0.5200 & 4 & 0.7857 & 0.6958 \\
\hline HESR049 & 3 & 0.3571 & 0.4206 & 2 & 0.2308 & 0.2123 & 1 & 0.0000 & 0.0000 \\
\hline HESR050 & 3 & 0.2143 & 0.4550 & 2 & 0.0000 & 0.3692 & 1 & 0.0000 & 0.0000 \\
\hline HESR051 & 3 & 0.4286 & 0.6376 & 3 & 0.0769 & 0.6923 & 3 & 0.1429 & 0.6640 \\
\hline HESR053 & 2 & 0.3571 & 0.4524 & 3 & 0.1538 & 0.5815 & 3 & 0.2143 & 0.3148 \\
\hline HESR054 & 3 & 0.0714 & 0.3148 & 2 & 0.0000 & 0.3692 & 2 & 0.0714 & 0.1984 \\
\hline HESR055 & 2 & 0.2143 & 0.3889 & 2 & 0.3077 & 0.2708 & 2 & 0.1429 & 0.2540 \\
\hline
\end{tabular}

$N_{\mathrm{A}}=$ number of alleles; $H_{\mathrm{E}}=$ expected heterozygosity; $H_{\mathrm{O}}=$ observed heterozygosity; $\mathrm{N}=$ individuals of population; $\mathrm{AC}=$ Acre; $\mathrm{RO}=$ Rondônia; $\mathrm{MT}=$ Mato Grosso.

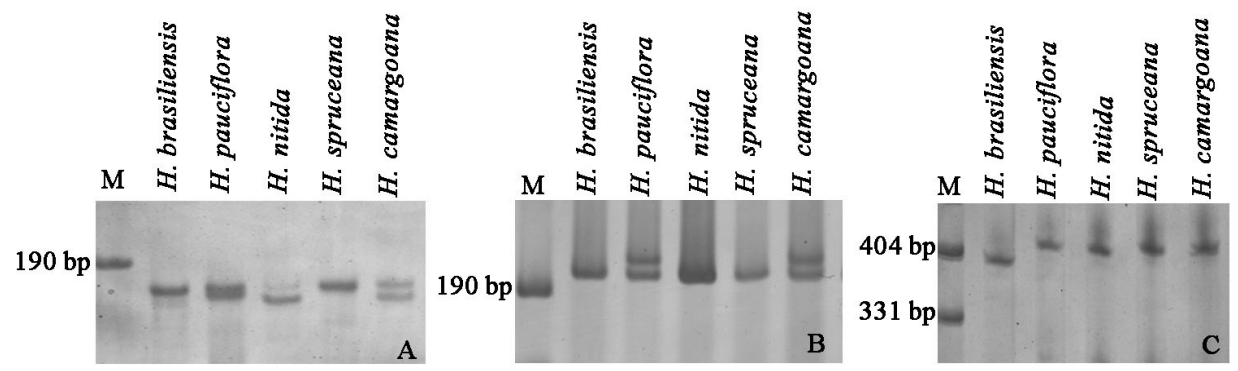

Figure 1. PCR amplification patterns showing polymorphism and transferability of EST-SSRs. A. Primer HESR030 amplification pattern in 5 species. B. Primer HESR045 amplification pattern in 5 species. C. Primer HESR050 amplification pattern in 5 species. Lane $M=$ a pUC19 DNA/MspI (HpaII) marker.

\section{ACKNOWLEDGMENTS}

Research partially supported by the National Natural Science Foundation of China (\#30860221, \#31271796), the Fundamental Research Funds of the Rubber Research Institute, CATAS (\#1630022011002), and the Earmarked Fund for Modern Agro-Industry Technology Research System (\#CARS-34).

\section{REFERENCES}

Altschul SF, Madden TL, Schaffer AA, Zhang J, et al. (1997). Gapped BLAST and PSI-BLAST: a new generation of protein database search programs. Nucleic Acids Res. 25: 3389-3402. 
An ZW and Huang HS (2005). A method for genomic DNA extraction from leaves of rubber tree (Hevea brasiliensis Müell. Arg.). Plant Physiol. Commun. 41: 513-515.

An ZW, Xie LL, Cheng H, Zhou Y, et al. (2009). A silver staining procedure for nucleic acids in polyacrylamide gels without fixation and pretreatment. Anal. Biochem. 391: 77-79.

Feng SP, Li WG, Huang HS, Wang JY, et al. (2009). Development, characterization and cross-species/genera transferability of EST-SSR markers for rubber tree (Hevea brasiliensis). Mol. Breed. 23: 85-97.

Huang X and Madan A (1999). CAP3: A DNA sequence assembly program. Genome Res. 9: 868-877.

Li D, Deng Z, Qin B, Liu X, et al. (2012). De novo assembly and characterization of bark transcriptome using Illumina sequencing and development of EST-SSR markers in rubber tree (Hevea brasiliensis Muell. Arg.). BMC Genomics 13: 192.

Priyadarshan PM and Gonçalves PS (2003). Hevea gene pool for breeding. Genet. Resour. Crop Evol. 50: 101-114.

Tan H (1987). Strategies in Rubber Tree Breeding. In: Improving Vegetatively Propagated Crops (Abbott AJ and Atkin RK, eds.). Academic Press, London, 27-62.

Temnykh S, DeClerck G, Lukashova A, Lipovich L, et al. (2001). Computational and experimental analysis of microsatellites in rice (Oryza sativa L.): frequency, length variation, transposon associations, and genetic marker potential. Genome Res. 11: 1441-1452.

Triwitayakorn K, Chatkulkawin P, Kanjanawattanawong S, Sraphet S, et al. (2011). Transcriptome sequencing of Hevea brasiliensis for development of microsatellite markers and construction of a genetic linkage map. DNA Res. 18: 471-482.

Yeh FC and Boyle TJB (1997). Population genetic analysis of co-dominant and dominant markers and quantitative traits. Belgian J. Bot. 129: 157. 